\title{
REMOVABLE SINGULARITIES IN THE BOUNDARY CONDITIONS
}

\author{
YURI V. EGOROV \\ Department of Mathematics, Moscow State University \\ Moscow V-234, 119899 Russia
}

1. Let $G$ be an open set in $\mathbb{R}^{n}$ and let $F$ be its boundary. Let $\Gamma$ be some part of $F$ which is a smooth $(n-1)$-dimensional submanifold. Let $A$ be a closed subset of $\Gamma$.

Let $u$ be a function harmonic in $G$ satisfying the boundary condition $D_{v} u=0$ on $\Gamma \backslash A$, where $v$ is the outer normal to $\Gamma$. When can we say that $D_{v} u=0$ on $\Gamma$, i.e. when the singularity of $u$ on $A$ is removable? It is evident that the answer depends on the structure of $A$ and on the behaviour of $u$ in a neighbourhood of $A$. For instance, if $A$ is a single point, then the singularity is removable if $|u(x)|=o\left(r^{2-n}\right)$ as $r \rightarrow 0$, where $r$ is the distance from $A$, and can be nonremovable if $n>2$ and $|u(x)|=O\left(r^{2-n}\right)$.

Indeed, let $f \in C_{0}^{\infty}(\Gamma)$. We show that if $|u(x)|=o\left(r^{2-n}\right)$, then

$$
\int_{\Gamma} f(x) D_{v} u(x) d S=0 .
$$

Let $A$ be the origin. Let $h \in C_{0}^{\infty}(\Gamma), h(A)=1$. Then

$$
\int_{\Gamma} f(x) D_{v} u(x) d S=\int_{\Gamma} f(x) D_{v} u(x) h(x / \varepsilon) d S
$$

for any $\varepsilon>0$. We can extend $f$ and $h$ in such a way that they vanish outside some neighbourhood of $A$ and $D_{v} f=D_{v} h=0$ on $\Gamma$. By the Green formula we have

$$
\int_{\Gamma} f(x) D_{v} u(x) h(x / \varepsilon) d S=\int_{G}(f(x) h(x / \varepsilon) \Delta u(x)-u(x) \Delta(f(x) h(x / \varepsilon))) d x
$$


and therefore,

$$
\int_{\Gamma} f(x) D_{v} u(x) d S=-\lim _{\varepsilon \rightarrow 0} \int_{G} u(x) \Delta(f(x) h(x / \varepsilon)) d x .
$$

It is clear that $|\Delta(f(x) h(x / \varepsilon))| \leq C \varepsilon^{-2}$. Therefore from the condition $|u(x)|=$ $o\left(r^{2-n}\right)$ it follows that $\int f(x) D_{v} u(x) d S=0$. The same is true if $u \in L_{p, \text { loc }}(G)$, where $p=n /(n-2), n>2$. This can be seen immediately if we apply Hölder's inequality.

On the other hand, if $\Gamma$ coincides locally with the plane $x_{n}=0$, then for the fundamental solution $E(x)$ of the Laplace operator we have $D_{n} E(x)=\delta\left(x^{\prime}\right)$ when $x_{n}=0$, where $x^{\prime}=\left(x_{1}, \ldots, x_{n-1}\right)$, and we can see that the singularity of the solution is non-removable if $n>2$ and $|u(x)|=O\left(r^{2-n}\right)$.

2. Now let $P(x, D)$ be a linear differential operator of order $m$ with coefficients smooth in $\bar{G}$, and suppose that another differential operator $B(x, D)$, which also has smooth coefficients, is defined on $\Gamma$. We do not make any assumptions about the type of the operator $P$.

Consider the following problem: when from the conditions: $P u=0$ in $G$, $B u=0$ on $\Gamma \backslash A$ does it follow that $B u=0$ on $\Gamma$ ? We state a number of sufficient conditions. All these conditions are sharp, which can be shown by suitable examples.

Our results can be easily transferred to boundary-value problems for linear systems of differential equations. The conditions on the smoothness of the coefficients of the operators $P$ and $B$, and on the smoothness of the manifold $\Gamma$ can, of course, be made essentially weaker.

3. Our main assumption is the validity of the Green formula:

$$
\int_{G}\left(P u \cdot v-u \cdot P^{\prime} v\right) d x=\int_{\Gamma} \sum_{j=1}^{N} B_{j}(x, D) u \cdot S_{j}(x, D) v d S
$$

for smooth functions $u$ and $v$, if $v=0$ in a neighbourhood of $F \backslash \Gamma$. Here $P^{\prime}$ is the operator transposed to $P, B_{j}$ and $S_{j}$ are differential operators with smooth coefficients, and one of the $B_{j}$, say $B_{1}$, coincides with the original operator $B$.

Assume also that

$$
S_{1}(x, D)=Q(x, D) D_{v}^{k}+S_{1}^{\prime}(x, D),
$$

where $D_{v}$ is differentiation in the normal direction, $Q$ acts in the directions tangent to $\Gamma$, and $k$ is some number, $0 \leq k \leq m-1$.

Suppose that the operators $S_{1}^{\prime}, S_{2}, \ldots, S_{N}$ do not involve the derivative $D_{v}^{k}$ (but they can involve $D_{v}^{i}$ for $i<k$ and for $i>k$ ) and that the equation $Q w=g$ has a solution $w \in C^{m}(\Gamma)$ for a set $M$ of functions $g$, which is dense in $C_{0}^{\infty}(\Gamma)$. 
4. TheOREm 1. Let $A$ be a single point. If $u(x)=o\left(r^{m-n-k}\right)$, where $r$ is the distance of $x$ from $A$, then $B u=0$ on $\Gamma$.

If $A$ is an infinite set, it is convenient to apply the Hausdorff measure for its description. The $d$-dimensional Hausdorff measure of $A$, denoted by $H_{d}(A)$, is defined as $\lim _{\varepsilon \rightarrow 0} \inf \sum r_{j}^{d}$, where the infimum is taken over all coverings of $A$ by countable collections of balls with radii $r_{j} \leq \varepsilon$.

5. Theorem 2. Let $-\infty<l<m, 1<p<\infty, 1 / p+1 / q=1$. If $P u=0$ in $G, B u=0$ on $\Gamma \backslash A, u \in W_{p}^{l}(G)$ and $H_{n-q(m-k-l)}(A)<\infty$, then $B u=0$ on $\Gamma$. If $u \in W_{\infty}^{l}(G)$, then the same is true if $H_{n-m+k+l}(A)=0$.

Here $W_{p}^{0}(G)=L_{p}(G)$ and $W_{p}^{l}(G)$ for $l$ natural is the space of functions whose derivatives of orders $\leq l$ are in $L_{p}(G)$. For negative integers $l$ this space consists of distributions of the form $\sum D^{i} f_{i}$ for $|i| \leq-l, f_{i} \in L_{p}(G)$.

6. Theorem 3. Let $P u=0$ in $G, B u=0$ on $\Gamma \backslash A$ and $u \in C^{l}(G \cup \Gamma)$. Assume that the order of the operator $B$ is greater than l. If $H_{n-m+k+l}(A)=0$, then $B u=0$ on $\Gamma$.

Here the space $C^{l}(M)$ for $l$ natural consists of functions whose derivatives of orders $\leq l-1$ are continuous and satisfy the Lipschitz condition in $M$. If $l>0$ is not an integer, then this is a space of functions whose derivatives of orders $\leq[l]$ satisfy the Hölder condition with exponent $l-[l]$ (here $[l]$ is the integer part of $l$ ). Finally, if $l \leq 0$, then $C^{l}(M)$ consists of distributions of the form $\sum D^{i} f_{i}$, where $|i| \leq-[l], f_{i} \in C^{[l]-l}(M)$.

7. The results for the Neumann problem, stated in the first section, are not sharp in the case $n=2$. It is well known that in this case the condition on $u$ must have the form $|u(x)|=o(\ln r)$. We state a similar sharp result for a general elliptic boundary-value problem.

Let $A$ be a smooth submanifold in $\Gamma$ of dimension $d=n-m$. Suppose $z_{1}, \ldots, z_{d}$ are local coordinates on $A$, and $y_{1}, \ldots, y_{n-d}$ are coordinates in the complementary space, so that the $y_{n-d}=x_{n}$ axis is transversal to $\Gamma$ and $y_{1}, \ldots, y_{n-d-1}$ are the inner coordinates in $\Gamma$.

Assume that $m=2 k$ and the operators $P, B_{1}, \ldots, B_{k}$ define a regular elliptic problem. Assume also that $m_{1}<m_{2}<\ldots<m_{k}=m-1$, where $m_{j}$ is the order of $B_{j}$. By the construction of the parametrix of this problem (see [1]),

$$
u(x)=Q P u+\sum_{j=1}^{k} Q_{j} B_{j}\left[u \otimes \delta\left(x_{n}\right)\right]+T u,
$$

where $Q, Q_{j}, T$ are pseudo-differential operators of orders $-m,-m_{j},-1$, respectively. Let $Q_{0}$ be the operator with symbol $1 / p_{0}(x, \xi)$ and $g_{j}=B_{j} u-B_{j} Q_{0} u$. Let $x \in \Gamma$. Let $r_{1}, \ldots, r_{k}$ be the roots of the equation $p_{0}\left(x, \xi^{\prime}, r\right)=0$ with positive 
imaginary parts. Let $R\left(x, \xi^{\prime}\right)=B\left(x, \xi^{\prime}\right)^{-1}$ where $B$ is the matrix with elements $b_{j l}\left(x, \xi^{\prime}\right)=b_{j}\left(x \cdot \xi^{\prime}, r_{l}\left(x, \xi^{\prime}\right)\right)$. Then

$$
\begin{aligned}
u(x)= & Q_{0} P u \\
& +(2 \pi)^{-n+1} \int \sum_{j, l=1}^{k} r_{j l}\left(x, \xi^{\prime}\right) F\left[g_{l}\right]\left(\xi^{\prime}\right) \exp \left(i r_{j}\left(x, \xi^{\prime}\right) x_{n}+i x^{\prime} \xi^{\prime}\right) d \xi^{\prime}+T u
\end{aligned}
$$

where $F[g]$ is the Fourier transform of $g$. Therefore the principal symbol of $Q_{j}$ is

$$
\sum_{l} r_{l j}\left(x, \xi^{\prime}\right) \exp \left(i r_{j}\left(x, \xi^{\prime}\right) x_{n}\right)
$$

and the order of homogeneity of $r_{l j}$ in $\xi^{\prime}$ is $-m_{j}$. Let

$$
r(y, z, \eta, \zeta)=r\left(x, \xi^{\prime}\right)=\sum r_{j l}\left(x, \xi^{\prime}\right) .
$$

The order of this function in $\xi^{\prime}$ is $1-m$.

Theorem 4. Let $P u=0$ in $G$ and $B_{j} u=0$ on $\Gamma \backslash A$ for $j=1, \ldots, k$, and $|u(x)|=o(\ln r)$, where $r$ is the distance of $x$ from A. Let

$$
\int_{|\eta|=1} r(0, z, \eta, 0) d S_{\eta} \neq 0 \quad \text { for } z \in A .
$$

Then $B_{j} u=0$ on $\Gamma$ and $u \in C^{\infty}(G \cup \Gamma)$.

The proof is based on a construction from [3].

\section{References}

[1] Yu. V. Egorov, Linear Differential Equations of Principal Type, Plenum, 1986.

[2] - On the removable singularities in the boundary conditions for differential equations, Vestnik Moskov. Univ. Ser. I Mat. Mekh. 1985 (6), 30-36 (in Russian).

[3] R. Harvey and J. Polking, Removable singularities of solutions of linear partial differential equations, Acta Math. 125 (1970), 39-56. 